\title{
Count-sampling circuits for heart-rate conditioning and other applications
}

\author{
ROBERT ADAMSON \\ Florida A tlantic University, Boca Raton, Florida 33432
}

\begin{abstract}
Simple count-sampling circuits are described for the study of heart-rate conditioning and other applications. The configurations are relatively inexpensive and easy to build.
\end{abstract}

The following circuits enable $E$ to preset sample intervals and critical response rates (e.g., heart rates). If the criterion is met within the interval, reinforcement follows; otherwise its possibility is lost until the next sample interval. The circuits allow for changes in interval duration, rate criterion, or both, at E's discretion. The configurations are relatively inexpensive and simple to complete.

The first circuit (Fig. 1) concerns the case in which heart rate must exceed criterion for reinforcement to be delivered. The amplified heart signal is obtained by the procedure described by Miller and DiCara (1967). It drives a sensitive relay (such as Sigma F-10000-S/SIL) so that each signal activates, through the relay contacts, the operate switch of an input modifier, $\mathrm{CX}_{1} .1$

The five flip-flops (FF 206) in Fig. 1 are connected as a 32-unit binary counter with selector switch outputs enabling any count from 0 to 31 to go into the AND-gate (AG 205). As shown, Binary Outputs 1, 4, 8, and 16 are $O N$, thus activating the rate input to $A G$ on a count of 29. This constitutes the response portion of the circuit (a more economical and less cumbersome binary counter is suggested later).

The interval-count match is accomplished when all inputs to $A G$ are $O N$. (Count inputs have been described.) The time interval input is initiated by a pulse delivered to the relay shown in the lower left of the circuit. The SPDT switch is part of a tape programmer (Gerbrands, Model PT-3A). The switch is wired so that, when it is in the NC position, a capacitor (150-200 microF) is charged. When the switch is in the NO position, the capacitor pulses the relay. (Any general-purpose dc relay may be used, e.g., Allied
KN 110.) Pulse duration is not critical; however, if more precise pulse control is desired, it may be accomplished by placing a variable resistor (about $1 \mathrm{~K}$ ohms) across the relay coil.

Action of the relay closes two sets of contacts, one of which resets the binary count to zero through the diode - reset input of $\mathrm{CX}_{1}$. The other closure delivers a pulse from $\mathrm{CX}_{2}$ to a one-shot (OS 204), which delivers a pulse of predetermined duration when triggered. Thus, the duration of the sample interval depends on the setting of OS, up to $30 \mathrm{sec}$ in OS 204. The OS output goes to AG together with the counter outputs. If all are $\mathrm{ON}$, i.e., the count has reached criterion within the interval, an output pulse is delivered from $\mathrm{AG}$ to a relay driver (RD 206), which, in turn, operates a relay that delivers reinforcement. For short sample intervals, a pulse limiter on the reinforcer is unnecessary. However, it is generally desirable and may be accomplished by using an OS set for a short pulse between $A G$ and $R D$. This is shown in all subsequent figures.

Additionally, the OS interval must be related realistically to the expected count, so that only one criterion count may be attained during the interval.

The FF binary counter of Fig. 1 illustrates the mechanism with which binary counts are generated. In practice, a more desirable configuration utilizes decade counters (DC 201). These have separate outputs for binary counts $1,2,4$, and 8 , and a transfer pulse on count 10 ( $\overline{8}$ output $)$, which activates a second $(10-\mathrm{min}$ count) counter. Thus, two decade counters will generate a count of $0-99$, with a total of eight binary outputs.

Figure 2 shows two linked decade counters, their outputs leading through toggle switches to an AG. This

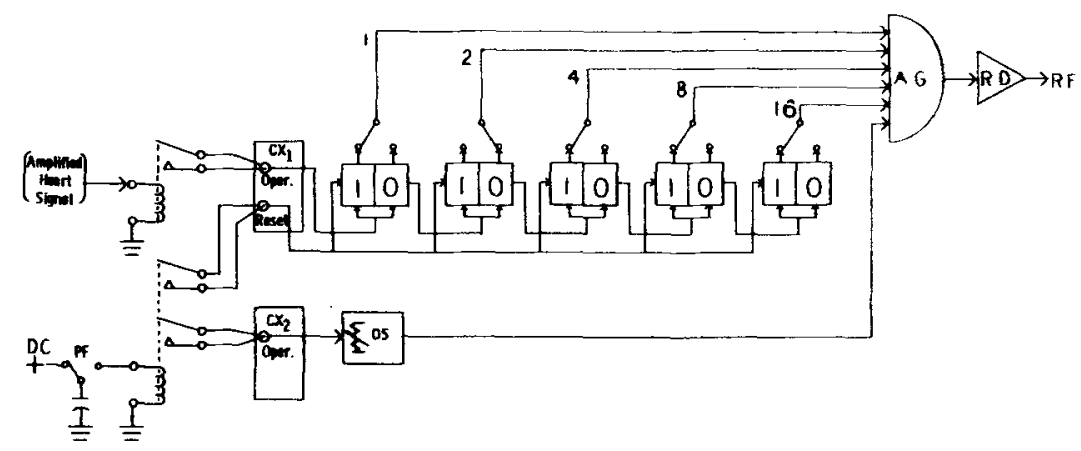

Fig. 1. A circuit in which a heart rate equal to or greater than a criterion set by $E$ is reinforced. 


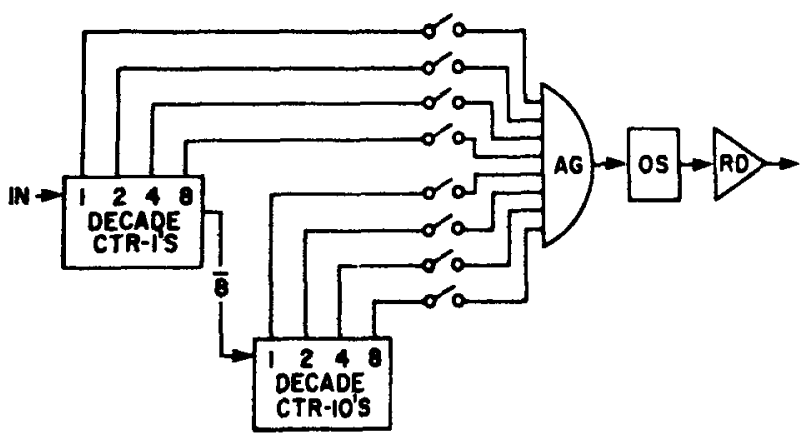

Fig. 2. Use of decade counters (DC) and a switch-bank to an AND-gate (AG) for setting response criteria.

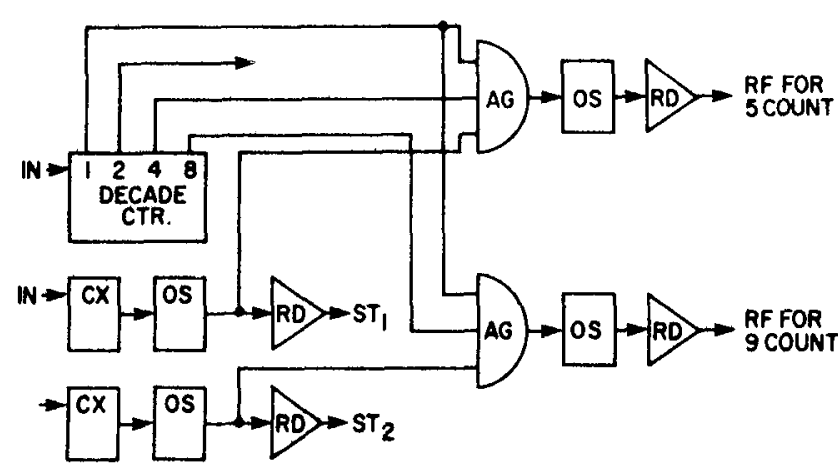

Fig. 3. A circuit for reinforcing different response rates to different stimulus presentations.

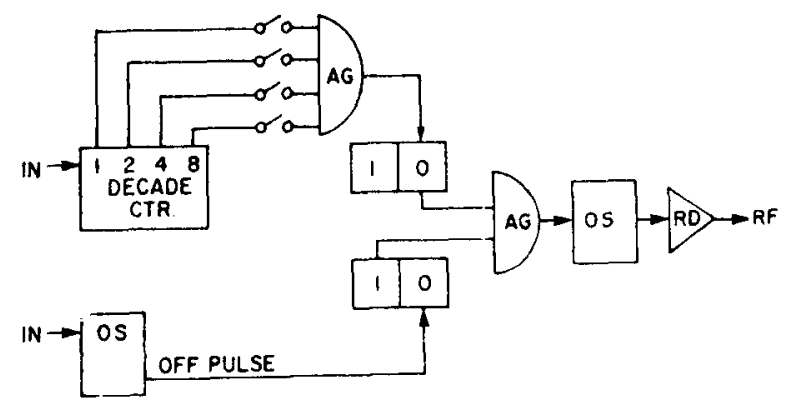

Fig. 4. A circuit in which a response rate less than a criterion set by $E$ is reinforced.

arrangement enables $E$, by switching in any binary combination, to set the criterion.
An extension of the circuit allows for conditioning different response rates (e.g., of pecking, barpressing) to different stimuli. Figure 3 shows the circuit, in which the binary outputs of the decade counter are coupled differentially in AGs 1 and 2 with outputs from the OS units, depending upon which has been triggered. Each OS output also controls a stimulus presentation, the stimulus, $\mathrm{ST}_{1}$ or $\mathrm{ST}_{2}$, being on for the interval. In Fig. 3, a response count of 5 during presentation of $\mathrm{ST}_{1}$ delivers reinforcement; during presentation of $\mathrm{ST}_{2}, 9$ responses would be required.

Thus far, attention has been directed to cases in which the rate must equal or exceed criterion. Figure 4 shows a schema for the situation in which the count during the sample interval must be less than criterion, e.g., in conditioning decreasing heart rate. The circuit shown is a comparator, in which $\mathrm{E}$ switches in the binary output desired from the decade counter to the upper AG. In the event this count is reached, the AG output switches the top FF to the inoperative " 1 " state, so that, in effect, reaching the criterion inactivates the remainder of the circuit.

As before, OS controls the interval. However, in this case, the "off" pulse of the OS triggered at the end of the interval is used. This pulse switches the lower FF to an operative " 1 " state, providing input to the right-hand AG. If the criterion count has not been reached at the end of the interval, the upper FF is still in the operative " 0 " state, and both inputs to AG are $\mathrm{ON}$. This provides an input to RD and delivers reinforcement.

Reset for decade counters and FFs is not shown in Figs. 3 and 4 , but is necessary for proper circuit function.

\section{REFERENCE}

Miller, N. E., \& DiCara, L. Instrumental learning of heart rate changes in curarized rats: Shaping, and specificity to discriminative stimulus. Journal of Comparative Physiological Psychology, 1967, 63, 12-19.

\section{NOTE}

1. BRS/LVE Module CX205. The parenthetical designations throughout refer to modules from the same manufacturer.

(Received for publication February 23, 1974; revision received April 2, 1974.) 